\title{
Spectral changes and BLR kinematics of eruptive changing-look AGN
}

\author{
M. W. Ochmann ${ }^{1}$, W. Kollatschny ${ }^{1}$ and M. Zetzl ${ }^{1}$ \\ Institut für Astrophysik, Universität Göttingen, Friedrich-Hund-Platz 1, 37077 \\ Göttingen, Germany, (E-mail: mochmann@astro.physik.uni-goettingen.de)
}

Received: August 15, 2019; Accepted: October 4, 2019

\begin{abstract}
We present results of recent optical spectroscopic variability campaigns for HE 1136-2304 and NGC 1566 which exhibited X-ray outbursts in July 2014 and June 2018, respectively. We show that the broad line region (BLR) structure in the changing-look AGN HE 1136-2304 is similar to that of non-changing-look AGN and that the BLR kinematics is dominated by Keplerian accretion disk (AD) rotation. Furthermore, we present an optical spectrum of NGC 1566 taken only a few weeks after its X-ray outburst, clearly showing drastic spectral changes.
\end{abstract}

Key words: active galactic nuclei - changing-look - broad line region - accretion disk - spectroscopy - HE 1136-2304 - NGC 1566

\section{Introduction - The changing-look phenomenon}

Active galactic nuclei (AGN) are known to exhibit variability in several frequency regimes. However, despite the mostly stochastic variations in the flux of AGN, their spectral type generally does not change. This is not true for optical changing-look (CL) AGN which exhibit signficant spectral changes leading to a change of the source's classification (e.g. from Sy-2 to Sy-1 or vice versa). Interestingly, these changes may happen on time scales of weeks to months and are often accompanied by outburts in several frequency regimes. Up to now a few dozen examples of CL AGN have been found. Very early observations of this phenomenon include - amongst others - NGC 351 (Collin-Souffrin et al., 1973), NGC4151 (Penston \& Perez, 1984) and Fairall 9 (Kollatschny \& Fricke, 1985). However, despite increasing interest in this topic, the cause for the changinglook phenomenon is still unknown. Possible explanations include accretion disk instabilities, tidal disruption events and a change of obscuration. Therefore, line profile studies of these eruptive spectral changes offer unique insight into AGN variability and allow - in combination with spectroscopic reverberation mapping - to determine the BLR structure and kinematics. 


\section{The changing-look AGN HE 1136-2304}

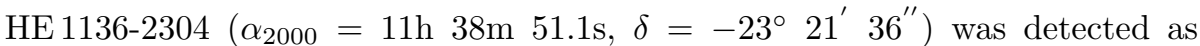
a variable X-ray source in 2014 (Parker et al., 2016). In comparison to the ROSAT survey in 1990, its $0.2-2 \mathrm{keV}$ flux increased by a factor of $\sim 30$. An optical spectroscopic follow-up observation revealed significant spectral changes, changing the classification of the galaxy. HE 1136-2304 is therefore classified as a changing-look AGN.

\subsection{Observations and Data Reduction}

The optical spectrum directly following the X-ray outburst was taken with the $10 \mathrm{~m}$ Southern African Large Telescope (SALT) (Parker et al., 2016) on July 7, 2014. To further investigate the variability behaviour of HE 1136-2304 we started a spectroscopic campaign with SALT on December 25, 2014, lasting until July 13, 2015. The mean cadence was $\sim 9$ days between February and July 2015. We reduced the spectra according to the standard procedure (bias and dark subtraction, flat-fielding, cosmic ray correction, illumination correction, wavelength calibration, night sky subtraction, and flux calibration). The resulting reduced spectra cover a spectral range of $4240 \AA-7040 \AA$ in the rest frame. As the flux of

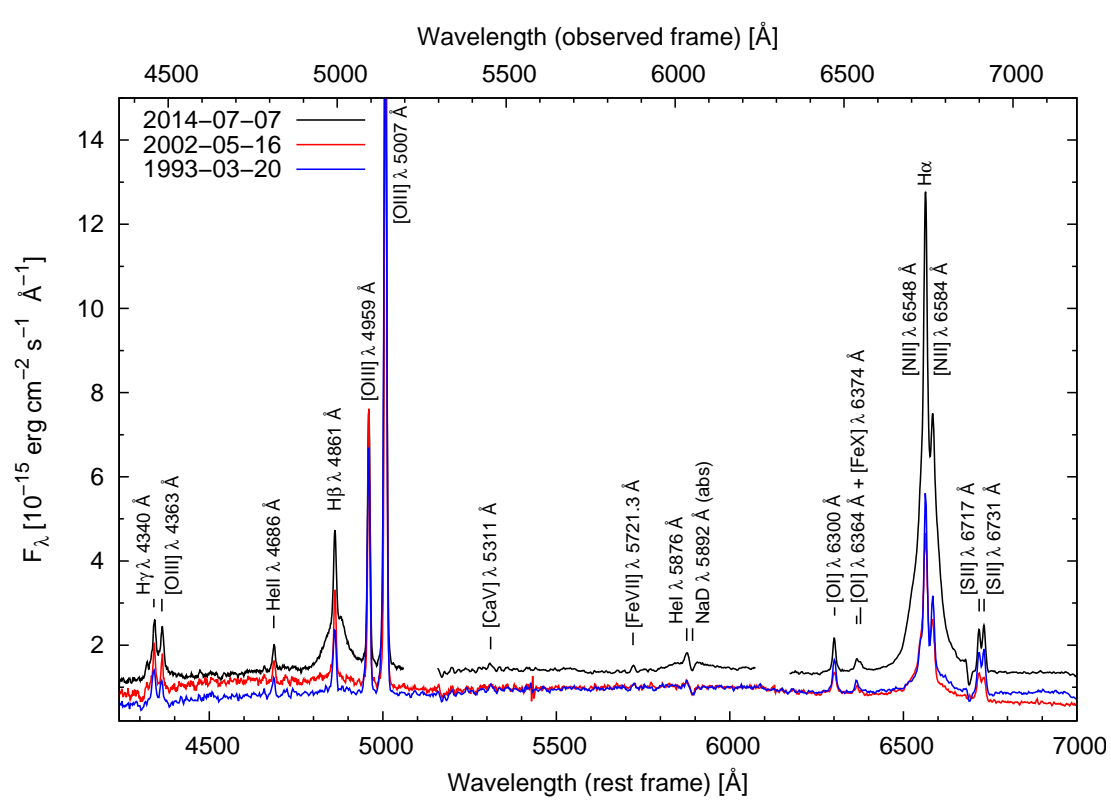

Figure 1. Optical spectra of HE 1136-2304 taken in 1993 (Hamburg/ESO), 2002 $(6 \mathrm{dF})$, and 2014. All spectra were calibrated to the absolute [OIII] $\lambda 5007$ flux from 1993-03-20. 
the narrow emission line [OIII] $\lambda 5007$ is treated to be constant on time scales of several years, the reduced spectra were calibrated to the absolute [OIII $] \lambda 5007$ flux given in Reimers et al. (1996). For further details on the instrumental setup and the data reduction we refer to Kollatschny et al. (2018).

\subsection{Results}

We show a comparison of optical spectra taken in 1993 (Hamburg/ESO survey; Reimers et al. (1996)), 2002 (6dF) and 2014 (Parker et al., 2016) in Fig. 1. In contrast to the spectra from 1993 and 2002, the post-outburst spectrum from 2014 especially shows strong and very broad $\mathrm{H} \alpha$ and $\mathrm{H} \beta$ lines with line widths of several thousand $\mathrm{km} \mathrm{s}^{-1}$ (FWHM). While HE 1136-2304's spectral classification corresponded to a Seyfert 1.9 type in 1993 and 2002, respectively, it changed its classification to a Seyfert 1.5 type after the X-ray outburst in 2014 (Zetzl et al., 2018).

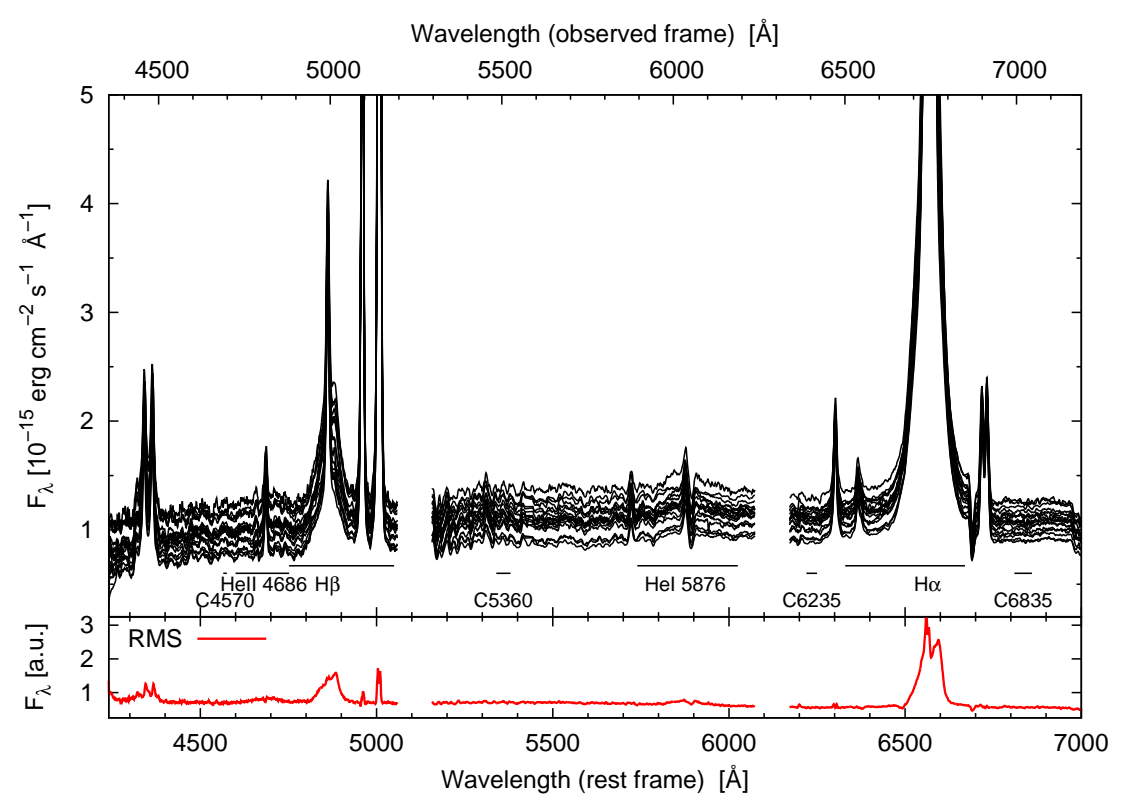

Figure 2. Finally reduced and intercalibrated optical spectra of the variability campaign of HE 1136-2304 with spectral ranges for light curve extraction (top). RMS spectrum of the variability campaign (bottom).

The finally reduced and calibrated spectra taken during our variability campaign and the resulting residual mean square (RMS) spectrum are shown in Fig. 2. Evidently, the spectrum of HE 1136-2304 exhibits clear variations of its continuum level. Furthermore, the RMS spectrum cleary shows variations not 
Table 1. Cross-correlation time lags with respect to the $4570 \AA$ continuum, FWHM (RMS), and line dispersion $\sigma$ (RMS) of the Balmer and Helium lines.

\begin{tabular}{lrcc}
\hline \hline Line & $\begin{array}{c}\tau \\
(\text { days })\end{array}$ & $\begin{array}{c}\text { FWHM (RMS) } \\
\left(\mathrm{km} \mathrm{s}^{-1}\right)\end{array}$ & $\begin{array}{c}\sigma(\mathrm{RMS}) \\
\left(\mathrm{km} \mathrm{s}^{-1}\right)\end{array}$ \\
\hline $\mathrm{H} \alpha$ & $15.0_{-3.8}^{+4.2}$ & $2668 \pm 150$ & $1816 \pm 150$ \\
$\mathrm{H} \beta$ & $7.5_{-5.7}^{+4.6}$ & $3791 \pm 150$ & $1767 \pm 150$ \\
He I $\lambda 5876$ & $7.3_{-4.4}^{+2.8}$ & $4131 \pm 400$ & $2098 \pm 400$ \\
He II $\lambda 4686$ & $3.0_{-3.7}^{+5.3}$ & $5328 \pm 500$ & $2962 \pm 500$ \\
\hline \hline
\end{tabular}

only in the broad Balmer lines but also - albeit lower - in the very broad higher ionization lines He I $\lambda 5876$ and He II $\lambda 4686$ (Kollatschny et al., 2018) ${ }^{1}$. In order to determine the time-lag between continuum and broad emission line variations - a method known as reverberation mapping - we extracted continuum and emission line light curves from all of the spectra. The corresponding spectral ranges for extraction are also shown in Fig. 2. To determine the integrated emission line flux, pseudo-continua to the left and right of each emission line were used to subtract the underlying continuum.

We present the resulting light curves of the continuum flux at $4570 \AA$ and $5360 \AA$, respectively, and the integrated fluxes of $\mathrm{H} \alpha, \mathrm{H} \beta, \mathrm{He} \mathrm{I} \lambda 5876$ and $\mathrm{He}$ II $\lambda 4686$ in Fig. 3. According to the notion of reverberation mapping, the emission line flux in the broad line region responds with a delay to the ionizing continuum considering that the continuum emission is generated close to the black hole. Hence, time delay measurements between the emission line and continuum light curves allow to determine the mean distance between the central ionizing source and the broad emission line region. Please note that the optical continuum can merely function as a surrogate for the ionizing X-ray and UV emission as it lags behind the X-ray and UV light curves (e.g. Zetzl et al., 2018). This additional delay has to be taken into account when evaluating the kinematics of the system (e.g. calculating the mass of the $\mathrm{SMBH}$ ).

The time-lags between the continua and emission lines were determined by correlating the continuum and emission line light curves. We used the interpolation cross-correlation function method (ICCF) developed by Gaskell \& Peterson (1987) and implemented in our own ICCF code (Dietrich \& Kollatschny, 1995). The time-lags were calculated using the centroids $\tau_{\text {cent }}$ of the ICCFs above $80 \%$ of the maximum correlation coefficient value $r_{\max }$. The resulting time-delays with respect to the continuum at $4570 \AA$ are given in Tab. 1 . The errors were

\footnotetext{
${ }^{1}$ Please note the variations in the [OIII] lines are entirely due to minor line profile variations during the campaign. The absolute [OIII] $\lambda 5007$ flux is constant.
} 


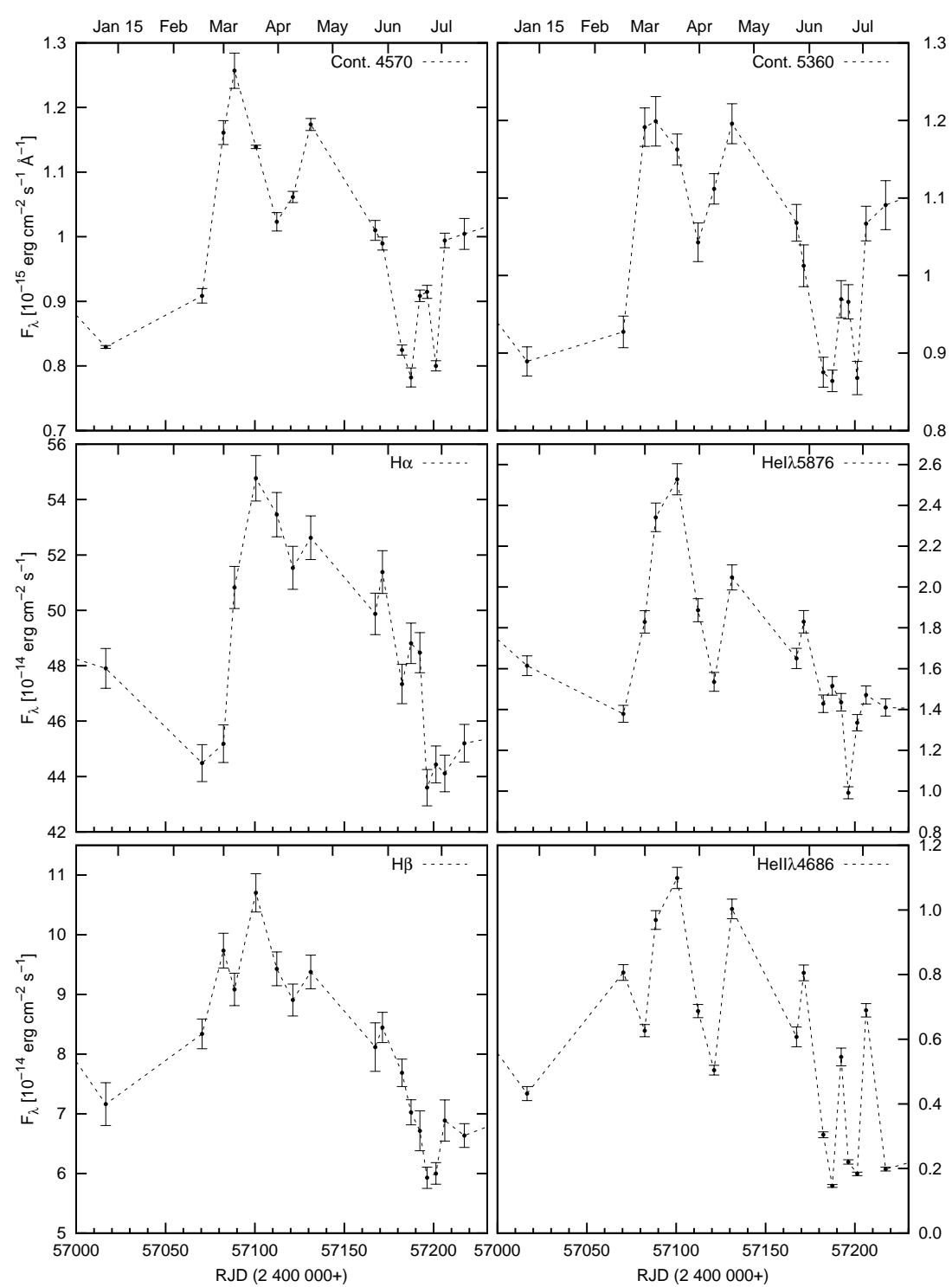

Figure 3. Light curves of the continua $4570 \AA$ and $5380 \AA$ as well as of the emission lines $\mathrm{H} \alpha, \mathrm{H} \beta, \mathrm{He} \mathrm{I} \lambda 5876$ and He II $\lambda 4686$ for the campaign between December 25 , 2014 and July 13, 2015.

estimated applying the flux redistribution/random subset selection (FR/RSS) method described in Peterson et al. (1998). 
The results indicate an ionization stratification of the BLR, i.e. the averaged $\mathrm{H} \alpha$ line originates the furthest away $\left(15.0_{-3.8}^{+4.2} \mathrm{~d}\right)$, the averaged $\mathrm{H} \beta$ line orginates at aprroximately half of this distance $\left(7.5_{-5.7}^{+4.6} \mathrm{~d}\right)$ while the Helium I and II line with higher ionization energy - originate closer inwards $\left(7.3_{-4.4}^{+2.8} \mathrm{~d}\right.$ and $3.0_{-3.7}^{+5.3} \mathrm{~d}$, respectively). In order to determine the vertical BLR structure we parametrized the line profiles by the ratio of their full-width at half maximum (FWHM) to their line dispersion $\sigma_{\text {line }}$. In this model the rotational line broadening of instrinsic Lorentzian profiles manifests as the FWHM while the intrinsic Lorentzian profiles are due to turbulent motions of the BLR gas (Kollatschny \& Zetzl, 2011, 2013a,b,c). To calculate the FWHM/ $\sigma$ ratios we measured the FWHM and line dispersion $\sigma$ in the RMS profile for each line. The values are presented in Tab. 1. The model calculations allow to determine the height of the line-emitting gas above the midplane for each line individually. The resulting cross-section of the BLR strucure is shown in Fig. 4. The black dot denotes the supermassive black hole $(\mathrm{SMBH})$ at the center of the AGN.

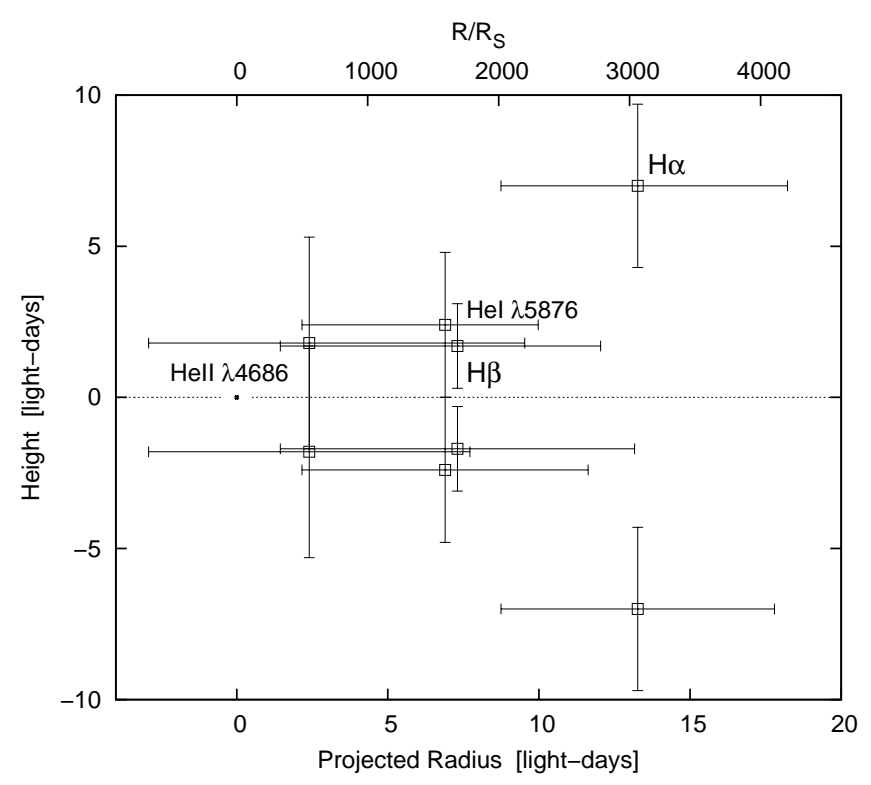

Figure 4. Cross-section of the line-averaged BLR structure in HE 1136-2304. The dot denotes the SMBH. The radius is given in both light-days and Schwarzschild radii of the SMBH in HE 1136-2304.

So far, we only analysed the line-averaged structure of the BLR. However, since the broadening of the lines is due to rotation, different parts of the line profile correspond to different rotational velocities and therefore distances. Particularly, we expect the outer line wings to originate closer to the SMBH than 
the line center. To investigate the profile variations in more detail we calculated the lags of individual line segments. We sliced the velocity profiles of the emission lines into segments with a width of $v=400 \mathrm{~km} \mathrm{~s}^{-1}$ and extracted the light curve for each individual segment. We then correlated these light curves with the $4570 \AA$ light curve (Kollatschny et al., 2018). The result is a $2 \mathrm{D}$ velocity delay map showing the time delay for each velocity line profile segment. The $2 \mathrm{D}$ velocity maps of $\mathrm{H} \alpha$ and $\mathrm{H} \beta$ are shown in Figs. 5 and 6 . The line centers show delays of $10-30 \mathrm{~d}$ whereas the line wings exhibit a faster response of $0-10 \mathrm{~d}$.

\section{NGC 1566 - a periodic changing-look AGN?}

NGC 1566 is a near $(z=0.005)$ face-on Seyfert galaxy which is known to have shown recurrent significant flux changes in several frequency bands. The outbursts have been accompanied by spectral changes, i.e. NGC 1566 changed from a nearly Sy-2 type classification in its low state to a nearly Sy-1 type classification after the outbursts (Parker et al. (2019) and references therein). In addition, the long-term light curves point towards a periodicity of the eruptive flux changes. A possible mechanism explaining the periodic outbursts was recently presented by Śniegowska \& Czerny (2019).

\subsection{Observations and Results}

We took an optical spectrum of NGC 1566 with SALT shortly after an X-ray outburst was detected in 2018 (Parker et al., 2019). This spectrum is shown in Fig. 7 together with an archival spectrum we took with the same instrument in 2012. The outburst spectrum shows a strong increase in continuum flux, especially towards the UV. Several lines now show strong broad components which have not been present when NGC 1566 was in its low state in 2012.

\section{Summary}

For the changing-look AGN HE 1136-2304, we were able to show an ionization stratification of the BLR similar to that of other AGN (e.g. Kollatschny et al., 2014). Furthermore, the vertical BRL structure is consistent with the bowlshaped geometry proposed by Goad et al. (2012), e.g. tested by Ramolla et al. (2018) for 3C 120 . The 2D velocity maps of the Balmer line profiles reveal a faster response of the line wings $(0-10 \mathrm{~d})$ in comparison to a slower response of the line centers $(10-30 \mathrm{~d})$. A similar behaviour is observed in the HeI $\lambda 5876$ and He II $\lambda 4686$ line (Kollatschny et al., 2018). The resulting kinematics is consistent with thin Keplerian disk BLR models (Horne et al., 2004). Overall, the BLR structure and kinematics of HE1136-2304 is similar to that of non-changing-look AGN.

For the changing-look AGN NGC 1566, our optical outburst spectrum from 2018 reveals drastic spectral changes in comparison to the low state spectrum 
from 2012. A thorough analysis of the outburst spectrum together with an archival spectroscopic campaign is in progress.

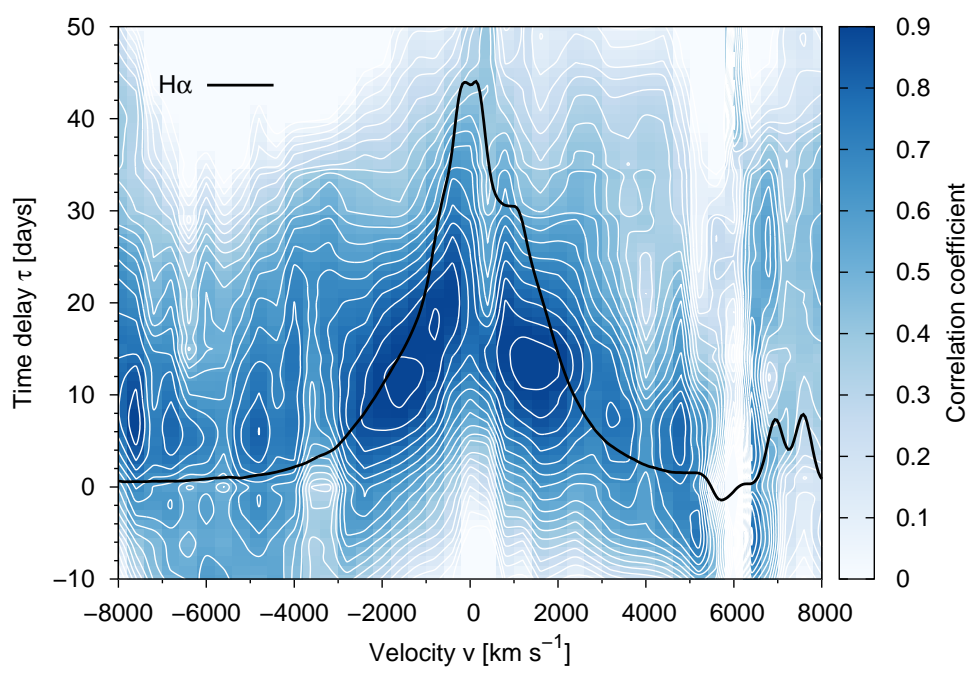

Figure 5. 2D velocity delay map of the $\mathrm{H} \alpha$ profile in HE 1136-2304 showing the correlation coefficient as a function of velocity and time delay (blue scale). The campaign-averaged line profile without narrow component is plotted in black.

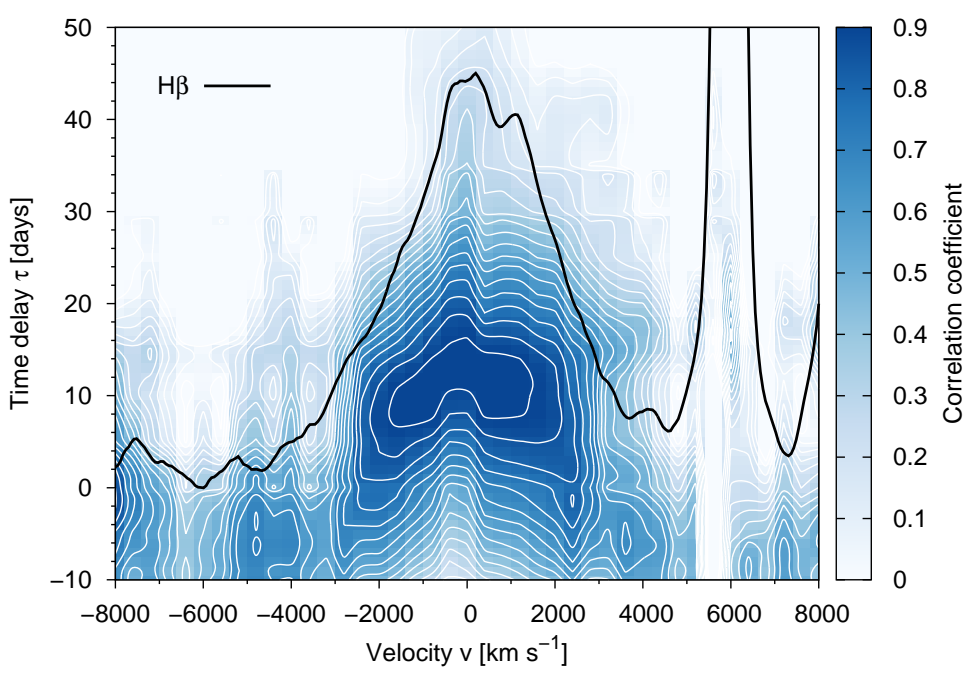

Figure 6. 2D velocity delay map of the $\mathrm{H} \beta$ profile in $\mathrm{HE} 1136-2304$ showing the correlation coefficient as a function of velocity and time delay (blue scale). The campaign-averaged line profile without narrow component is plotted in black. 


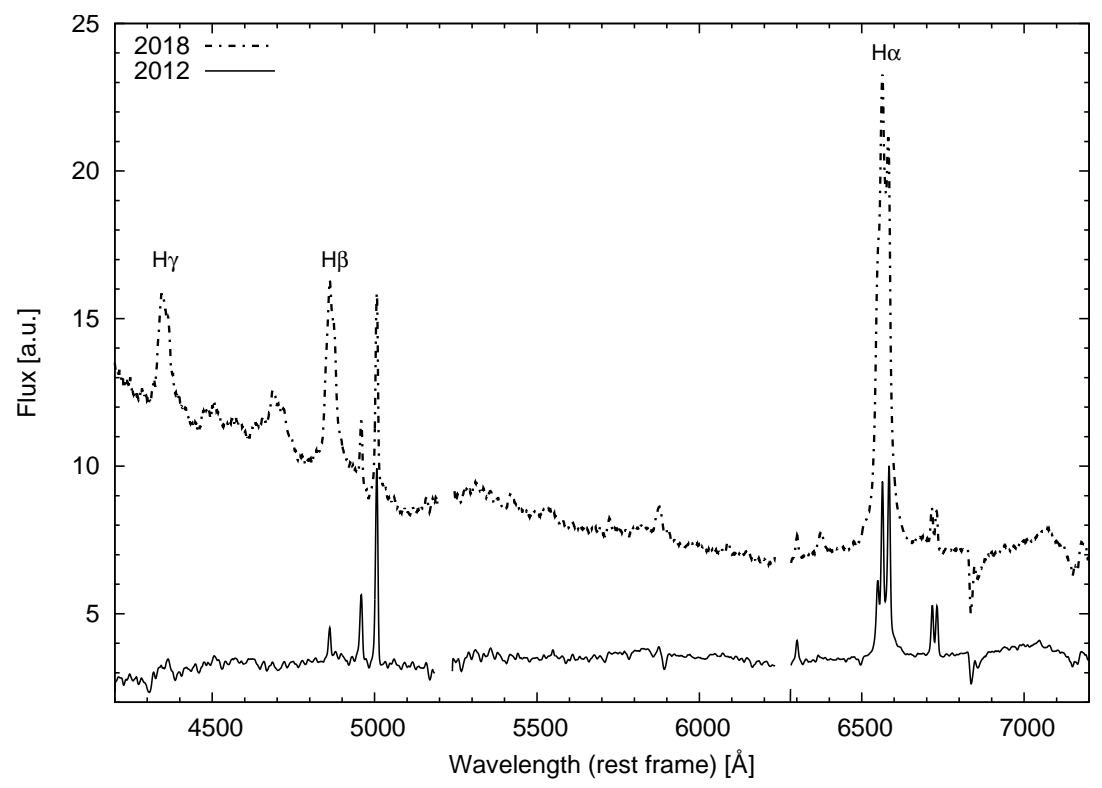

Figure 7. Optical calibrated spectra of NGC 1566 in its low state (2012) and in its high state after the outburst (2018). Both spectra were taken with SALT and reduced the same way as the spectra of HE 1136-2304.

Acknowledgements. This work has been supported by the DFG grant Ko 857/331 .

\section{References}

Collin-Souffrin, S., Alloin, D., \& Andrillat, Y., A discussion of the new variations observed in the nucleus of the Seyfert galaxy NGC 3516. 1973, Astron. Astrophys., $\mathbf{2 2}, 343$

Dietrich, M. \& Kollatschny, W., Optical and ultraviolet emission-line variability of NGC 5548. The coordinated UV and optical monitoring campaign of 1989. 1995, Astron. Astrophys., 303, 405

Gaskell, C. M. \& Peterson, B. M., The accuracy of cross-correlation estimates of quasar emission-line region sizes. 1987, Astrophys. J., Suppl., 65, 1

Goad, M. R., Korista, K. T., \& Ruff, A. J., The broad emission-line region: the confluence of the outer accretion disc with the inner edge of the dusty torus. 2012, Mon. Not. R. Astron. Soc., 426, 3086

Horne, K., Peterson, B. M., Collier, S. J., \& Netzer, H., Observational Requirements for High-Fidelity Reverberation Mapping. 2004, Publ. Astron. Soc. Pac., 116, 465 
Kollatschny, W. \& Fricke, K. J., The fading of the Seyfert galaxy F-9. 1985, Astron. Astrophys., 146, L11

Kollatschny, W., Ochmann, M. W., Zetzl, M., et al., Broad-line region structure and line profile variations in the changing look AGN HE 1136-2304. 2018, Astron. Astrophys., 619, A168

Kollatschny, W., Ulbrich, K., Zetzl, M., Kaspi, S., \& Haas, M., Broad-line region structure and kinematics in the radio galaxy 3C 120. 2014, Astron. Astrophys., 566, A106

Kollatschny, W. \& Zetzl, M., Broad-line active galactic nuclei rotate faster than narrow-line ones. 2011, Nature, 470, 366

Kollatschny, W. \& Zetzl, M., Accretion disk wind as explanation for the broad-line region structure in NGC 5548. 2013a, Astron. Astrophys., 551, L6

Kollatschny, W. \& Zetzl, M., The shape of broad-line profiles in active galactic nuclei. 2013b, Astron. Astrophys., 549, A100

Kollatschny, W. \& Zetzl, M., Vertical broad-line region structure in nearby active galactic nuclei. 2013c, Astron. Astrophys., 558, A26

Parker, M. L., Komossa, S., Kollatschny, W., et al., The detection and X-ray view of the changing look AGN HE 1136-2304. 2016, Mon. Not. R. Astron. Soc., 461, 1927

Parker, M. L., Schartel, N., Grupe, D., et al., X-ray spectra reveal the reawakening of the repeat changing-look AGN NGC 1566. 2019, Mon. Not. R. Astron. Soc., 483, L88

Penston, M. V. \& Perez, E., An evolutionary link between Seyfert I and II galaxies? 1984, Mon. Not. R. Astron. Soc., 211, 33P

Peterson, B. M., Wanders, I., Bertram, R., et al., Optical Continuum and EmissionLine Variability of Seyfert 1 Galaxies. 1998, Astrophys. J., 501, 82

Ramolla, M., Haas, M., Westhues, C., et al., Simultaneous $\mathrm{H} \alpha$ and dust reverberation mapping of 3C 120: Testing the bowl-shaped torus geometry. 2018, Astron. Astrophys., 620, A137

Reimers, D., Koehler, T., \& Wisotzki, L., The Hamburg/ESO survey for bright QSOs. II. Follow-up spectroscopy of 160 quasars and Seyferts. 1996, Astron. Astrophys., Suppl., 115, 235

Śniegowska, M. \& Czerny, B., Mechanism of the Changing Look phenomenon in Active Galactic Nuclei. 2019, arXiv, 1904.06767

Zetzl, M., Kollatschny, W., Ochmann, M. W., et al., Long-term optical, UV, and Xray continuum variations in the changing-look AGN HE 1136-2304. 2018, Astron. Astrophys., 618, A83 